\title{
Introduction to the Minitrack on Critical and Ethical Studies of Digital and Social Media
}

\author{
Amelia Acker \\ School of Information \\ University of Texas at Austin \\ aacker@ischool.utexas.edu
}

\author{
Brian Beaton \\ Center for Expressive Tech \\ Cal Poly (San Luis Obispo) \\ brbeaton@calpoly.edu
}

\author{
Lana Swartz \\ Department of Media Studies \\ University of Virginia \\ des5z@,virginia.edu
}

\begin{abstract}
This minitrack addresses the cultural and political impacts of digital and social media (DSM), interrogating how DSM fundamentally impacts how we consume, circulate, and make sense of networked information. Both papers contain two broad themes that will interest and inspire scholars of social media and systems science. First, the studies critically interrogate how and when digital and social media support existing power structures or realign power individuals connecting to groups and making community online. Second, both of these contemporary case studies raise awareness and illustrate the ethical issues associated with doing research on digital and social media in platforms that contain black boxes within black boxes. Conceptually and empirically grounded, each paper provides readers with new metaphors and ethical considerations for interpreting information exchange in networked communities, as well as key ideas and paradigms for the science of social media. By addressing the cultural and political impacts of DSM, both of these groundbreaking and original studies illustrate how the flow of data through digital and social media act to organize the social and political dimensions of these participatory information systems.
\end{abstract}

\section{Session 1}

\subsection{Cracking Open the Black Box of Genetic Ancestry Testing}

This paper reports on a study of forum discussions about genetic ancestry test results and databases on Stormfront, an online discussion forum for white nationalists. By examining the identity formation and boundary maintenance of personal genomic testing results, the authors highlight how the work of interpreting genetic testing, results, and ad-hoc genetic databases, is both highly social and scientifically tenuous on Stormfront, where debates over race and collective identity are established and maintained by community members. With online ethnographic methods, the authors carefully show how a "biosociality" emerges for Stormfront users when confronted with results they dislike and how they exert ownership and interpretation over their personal genetic tests. The authors show how white nationalists fashion a collective identity by 'moving their data' to reckon with biological markers, contentious indicators of racial phenotypic information and the black boxed algorithms that private testing platforms provide.

\section{2 The Ethics of Pyschometrics in Social Media: A Rawlsian Approach}

This paper examines targeted political and social media advertising based on psychometrics techniques by critiquing the motivations and ethical outcomes of their application in both commercial and democratic processes. Through a Rawlsian framework, the author compares how psychometrics influences the experience commercial advertising and political campaign influence on digital and social media platforms. After providing a brief and timely overview of the application of psychometrics in advertising, political persuasion, and the rise of social media campaigning, the author argues how Rawlsian principles are violated through expectations of transparency, misinformation, and uneven information access. The author argues how these different violations can impede upon a citizen's democratic duties-without access to pluralistic political discourse in social networks, social media campaigning has the potential to create non-trivial and broad experiences of information asymmetry and thus inhibiting free expression of political liberty because of hyper-targeted political and social media information. 